\title{
An Evaluation of the Therapeutic Nutrition Knowledge of
}

\section{Belizean Nurses.}

Marcia Magnus, $\mathrm{PhD}^{1 *}$ and Tabe Besong RN MSc ${ }^{1}$.

${ }^{1}$ Department of Dietetics and Nutrition, Florida International University, Miami, USA.

*Corresponding Author: Marcia Magnus, Department of Dietetics and Nutrition, Florida International University, Miami, USA.

Received date: February 06, 2021; Accepted date: February 12, 2021; Published date: February $16,2021$.

Citation: Marcia Magnus, Tabe Besong RN. An Evaluation of the Therapeutic Nutrition Knowledge of Belizean Nurses, J. New Medical Innovations and Research, 2(1): DOI: 10.31579/jnmir.2021/004.

Copyright: () 2021 Marcia Magnus and Tabe Besong RN. This is an open-access article distributed under the terms of The Creative Commons Attribution License, which permits unrestricted use, distribution, and reproduction in any medium, provided the original author and source are credited.

\begin{abstract}
In Belize, patients rely on nurses for nutritional guidance on non-communicable diseases in the Caribbean. The objectives of this study were to quantify therapeutic nutritional knowledge of 198 Belizean nurses using the AlShwaiyat questionnaire. The mean correct response rate for the therapeutic nutritional knowledge was suboptimal $(53.30 \% \pm 17.20)$. Nurses in the West region of the country had the highest mean knowledge scores on nutrition and diabetes $75.41 \pm 20.10, \mathrm{p}=0.003$; on nutrition and cardiovascular diseases $57.23 \pm 10.90, \mathrm{p}=0.000$; and in overall knowledge $62.34 \pm 9.76 \mathrm{p}=0.000$. Nurses without a diagnosis of diabetes had higher mean knowledge scores on a) nutrition and diabetes $63.86 \pm 26.07, \mathrm{p}=0.001$; b) nutrition and obesity questions $49.55 \pm 20.80$ $\mathrm{p}=0.004$; c) nutrition and cardiovascular disease $53.30 \pm 15.70, \mathrm{p}=0.007$ and $\mathrm{d}$ ), overall mean $55.24 \pm 16.67$, $\mathrm{p}=0.000$. There is the need to address the low levels of knowledge of Belizean nurses.
\end{abstract}

Keywords: nurses; therapeutic nutritional knowledge; Belize

\section{Introduction}

Belize is the only English-speaking country in Central America. In 2019, Belize had a population of 383,000 [1]. Belize is a developing uppermiddle-income country, with a GDP per capita of US\$ 4,829 in 2016. The 2009 Country Poverty Assessment revealed a poverty rate of $68 \%$ for the indigenous Maya, a child poverty rate of $50 \%$, and a national average poverty rate of $41.3 \%$ [2]. Belize, like other English-speaking Caribbean countries and Central American countries, has been experiencing an increased prevalence of non-communicable chronic diseases, many of which respond to dietary management. In 2015, the crude diabetes prevalence rate was $14.2 \%$, the highest of all counties in North America and the Caribbean region [3]. Between 2000 and 2016, adult male obesity increased from $9.7 \%$ to $16 \%$, in adult females from $23.0 \%$ to $31.5 \%$ [1]. Although diabetes prevalence increased from $11.3 \%$ to $15.2 \%$ among females, and from $7.8 \%$ to $10.0 \%$ among males, high blood pressure in adult males decreased from $27.5 \%$ to $24.4 \%$, and among females from $23.4 \%$ to $21.0 \%$ [1]. Between 2002 and 2014, the prevalence of female adult diabetes increased from $11.3 \%$ to $15.2 \%$, with male adult diabetes increasing from $7.8 \%$ to $10.0 \%$ [1]. The leading cause of death in people aged 50 and over was diabetes and its complications, followed by ischemic heart disease, hypertension, advanced HIV infection, and chronic liver disease and cirrhosis [2]. In 2019, progress against global nutrition targets for adult female and male obesity, adult female and male diabetes were described as "no progress or worsening" in Belize [1].

In 2015 , the over 60 -year old population accounted for $5.9 \%$ of the total population, and this segment of the population had a higher hospital utilization rate than the rest of the population [2]. Research from twelve countries demonstrated that $50.5 \%$ of older people in rehabilitation were malnourished, closely followed by older people in hospital $(38.7 \%)$ and then those in aged care facilities (13.8\%) [4]. Nurses' role in initial nutrition screening has been described as "crucial" in the identification of patients who are already malnourished and those who are at risk of becoming malnourished, with the goal of developing nutritional care strategies which prevent severe malnutrition [5].

Although between 2010 and 2015, the population density of physicians and dentists increased, the population density of nurses decreased from 14.5 in 2010 to 12.3 per 10,000 , largely due to migration [2]. In 2020 , eight nutritionists/dietitians were employed in seven public and three private hospitals, and 54 health clinics, serving a population of 383,000 persons [1].

US data indicate that nurses are 40 times more than dietitians, and 100 times more than certified diabetes educators to meet hospitalized diabetes patients on a daily basis [6]. Nurses play an important role in patients' nutritional care. Nurses often fill the role of nutrition counsellors by providing nutrition screening and/or nutrition advice to patients [7]. Because they have the most contact with patients, nurses play a key multidisciplinary role and often provide nutrition screening, referral, facilitation of recommendations to adjust a diet plan, and implementation of a special diet modification with patients, and family members [7]. Nurses play equally important roles which complement the role of the dietitian to ensure adequate nutrition for patients $[8,9]$.

Nurses' roles in nutritional support have been mandated by governmental regulations internationally. The Australia Ministry of Health states that nurses are responsible for monitoring and documenting nutritional intake [10]. The UK Ministry of Health decrees that nurses are expected to a) 
promote healthier lifestyle choices from the point of admission through to discharge, to conduct nutritional assessment, and b) offer appropriate lifestyle advice and an effective referral system which supports positive long-term behavioural change [11]. In the USA, nutrition has been described as the second most important area for nursing care. Nutrition topics such as nutritional assessment and monitoring, diet therapy, and enteral and parenteral nutrition have been included in the National Council Licensure Examination for Registered Nurses [12]. In Belize, there is no official Ministry of Health document which specifies the role of nurses in nutrition care. Based on a conversation with a $\mathrm{L}$ Castillo, RN, NP, (August, 2020), nurses are responsible for feeding patients via the oral, enteral and parenteral routes; breastfeeding education, and to provide holistic care.

Despite widespread calls for nurses' role in nutritional care, the Academy of Nutrition and Dietetics, the world's largest association of nutrition professionals, has identified patient risks which derive from "scope creep" - health care professionals who go beyond their scope of practice and engage in activity for which they are not qualified [13]. Although RDNs, nurses and pharmacists all deliver Diabetes Self-Management training to patients, when professionals who provide care for which they were not trained, scope creep can be dangerous and put patients and clients at risk of harm. The Academy believes that the provision of medical nutrition therapy and other complex dietetics and nutrition services should only be provided by individuals who have at minimum the specialized training and competencies of RDNs.

\section{Objective}

Because there are no published studies on the therapeutic nutrition knowledge of nurses in Belize, this study was undertaken. The objective of this study was to determine the extent to which the nurses have the nutrition knowledge needed to educate and improve the lives of persons living with obesity, diabetes and hypertension.

\section{Research questions}

1. What is the level of therapeutic nutrition knowledge among Belizean nurses?

2. How is nurses' knowledge affected by health region, gender, age group, education level, years of service; self-reported weight status, and diagnosed diabetes mellitus and hypertension?

Therapeutic nutrition knowledge was defined as the latest theories and research in nutrition and health sciences to individuals seeking to manage chronic disease or promote optimum health. Nurses' therapeutic nutrition knowledge has been reported as $35 \% \pm 3.8$ in South Africa [14], 60\% \pm 8.4 in Australia [15], 56.75\% \pm 10.84 in Turkey [16], 58.8\% \pm 13.2 in Jordan [17], 58.4\% in Korea [18], $44.9 \% \pm 3.17$ in Ghana [19], $65 \% \pm 11$ in the United States of America [20]. Given the low levels of nurses' nutrition knowledge, all authors have recommended better educational preparation in nutrition and specific treatment guidelines for nurses.

\section{Research design and setting}

Of the 635 nurses in Belize, 198 were randomly recruited to respond to the therapeutic nutrition knowledge questionnaire which included items on diabetes (5 items), obesity (9 items) and cardiovascular diseases (17 items). The correct response for each question was scored as [1]. Incorrect and "don't know" answers were scored as (0). Demographic factors (health region, gender, age, level of education, years of work experience), and self-reported weight status, diabetes, and hypertension diagnosis) were conceptualized based on the work of Al-Shwaiyat, 2013 [17].

\section{Sample Size and sample selection}

G-Power version 3.0.10 was used to determine the appropriate sample size of 198. This was considered sufficient to yield 0.80 power using Ftest with a significant level of 0.05 and effect size 0.13 . A convenience sample was derived. Inclusion criteria were registered nurses, practical nurses, nurses working in both clinics and hospitals. Excluded from the study were nurse specialists, nurse supervisors and nurses working at the private sector.

\section{Instrumentation}

The Al-Shwaiyat et al., 2013 instrument [17] was used. The first section of the instrument included demographic characteristics, and the second section had therapeutic nutrition knowledge questions--five diabetes questions, nine obesity questions, and 17 questions on cardiovascular diseases. It had a Cronbach's Alpha reliability coefficient of 0.709 . To assess the face validity of the instrument, a focus group of Belizean nurses was conducted to assess their understanding of questions and perception of the cultural appropriateness of the instrument. A pilot study was undertaken among five nurses to evaluate the time, quality and structure of the questions; and to also determine if there were any problems or misinterpretation of the questionnaire.

\section{Data collection}

Permission to conduct this study was granted by the Institution Review Board of Belize, and by hospital managers. The questionnaires were administered to nurses three hours before the end of their shifts. Nurses were advised of their right to refuse to participate even after giving consent. Privacy of nurses' information was maintained to prevent access by others who are not directly involved in the research. Questionnaires were coded to maximize respondents' confidentiality and anonymity.

\section{Data analysis}

Questionnaires were coded and analyzed with SPSS version 25.0 [21]. Analysis of Variance (ANOVA), t-test and Pearson correlation, and regression analysis were used to test the relationships between variables.

\section{Results}

Among 198 nurses, the majority were female (82.9\%), and $67.9 \%$ were registered nurses (Table 1). Nurses were almost evenly distributed among the four health regions of Belize. The largest percentage $(29.4 \%)$ had 6 10 years of work experience. For self-reported weight status, $44.1 \%$ described themselves as being at recommended weight, and $48.7 \%$ as overweight. Only $10.2 \%$ reported a diagnosis of diabetes mellitus status, and $16.8 \%$ reported having hypertension. 


\begin{tabular}{|c|c|c|}
\hline Demographic characteristic & $\mathrm{N}$ & $\%$ \\
\hline $\begin{array}{l}\text { Health region: } \\
\text { North } \\
\text { South } \\
\text { West } \\
\text { Central }\end{array}$ & $\begin{array}{l}47 \\
48 \\
48 \\
55\end{array}$ & $\begin{array}{l}23.7 \\
24.2 \\
24.2 \\
27.8\end{array}$ \\
\hline $\begin{array}{l}\text { Gender: } \\
\text { Male } \\
\text { Female }\end{array}$ & $\begin{array}{l}33 \\
160\end{array}$ & $\begin{array}{l}17.1 \\
82.9\end{array}$ \\
\hline $\begin{array}{l}\text { Age group (years): } \\
\leq 25 \\
31-35 \\
\geq 36\end{array}$ & $\begin{array}{l}36 \\
92 \\
69\end{array}$ & $\begin{array}{l}18.3 \\
46.7 \\
35.0\end{array}$ \\
\hline $\begin{array}{l}\text { Educational level: } \\
\text { LPN } \\
\text { RN }\end{array}$ & $\begin{array}{l}63 \\
133 \\
\end{array}$ & $\begin{array}{l}32.1 \\
67.9 \\
\end{array}$ \\
\hline $\begin{array}{l}\text { Years of work experience: } \\
\leq 5 \\
6-10 \\
11-15 \\
\geq 16\end{array}$ & $\begin{array}{l}55 \\
58 \\
33 \\
51\end{array}$ & $\begin{array}{l}27.9 \\
29.4 \\
16.8 \\
25.9\end{array}$ \\
\hline $\begin{array}{l}\text { Nurses' self-reported weight status: } \\
\text { At Recommended Weight } \\
\text { Overweight } \\
\text { Underweight }\end{array}$ & $\begin{array}{l}86 \\
95 \\
14\end{array}$ & $\begin{array}{l}44.1 \\
48.7 \\
7.2\end{array}$ \\
\hline $\begin{array}{l}\text { Self-reported diabetes diagnosis: } \\
\text { No DM diagnosis } \\
\text { DM diagnosis }\end{array}$ & $\begin{array}{l}176 \\
20\end{array}$ & $\begin{array}{l}89.8 \\
10.2\end{array}$ \\
\hline $\begin{array}{l}\text { Self-reported hypertension diagnosis: } \\
\text { No HBP diagnosis } \\
\text { HBP diagnosis }\end{array}$ & $\begin{array}{l}164 \\
33 \\
\end{array}$ & $\begin{array}{l}83.2 \\
16.8 \\
\end{array}$ \\
\hline
\end{tabular}

Table 1: Demographic characteristics of Belizean nurses $(\mathrm{N}=198)$

The mean therapeutic nutrition knowledge score of 198 Belizean nurses was 53.31\% \pm 17.20 (Table 2). The mean knowledge score for diabetes mellitus questions was $62.90 \% \pm 26.70,51.0 \% \pm 16.50$ for questions on cardiovascular diseases, and $47.00 \% \pm 20.90$ for obesity questions. Nurses in Belize's West region had a significantly higher overall mean knowledge score $62.34 \pm 9.76 \mathrm{p}=0.000$; and higher mean knowledge scores for nutrition and diabetes $75.41 \pm 20.10, p=0.003$; nutrition and cardiovascular diseases $57.23 \pm 10.90, p=0.000$; compared to nurses in other regions. Female nurses had a mean score of $54.58 \% \pm 16.76$, while males had a mean score of $53.50 \% \pm 16.59$. Nurses who were $>36$ years of age had the highest mean score of $57.80 \% \pm 14.45$, followed by those $\leq 25$ years $(54.28 \% \pm 17.84)$ and lastly, those $>31-35$ years $(50.57 \% \pm 18.11)$. Registered nurses had a higher score of $55.40 \% \pm 17.61$, while practical nurses had a score of $49.50 \% \pm 15.44$. Nurses with less than 5 years of work experience had the highest score $61.25 \% \pm 14.24$, followed by those with $\geq 16$ years $(56.05 \% \pm 15.50)$, those with $6-10$ years $(49.92 \% \pm 17.63)$ and lastly, those with $11-15$ years of work experience $(44.57 \% \pm 17.36)$.

\begin{tabular}{|c|c|c|c|c|c|}
\hline Variables & $\begin{array}{l}\text { Nurses } \\
\mathrm{N}(\%)\end{array}$ & $\begin{array}{l}\text { Nutrition and } \\
\text { diabetes } \\
\text { knowledge } \\
\text { Mean } \pm \text { SD }\end{array}$ & $\begin{array}{l}\text { Nutrition and } \\
\text { obesity } \\
\text { knowledge } \\
\text { Mean } \pm \text { SD }\end{array}$ & $\begin{array}{ll}\text { Nutrition } & \text { and } \\
\text { cardiovascular } & \\
\text { diseases } & \\
\text { Mean } \pm \text { SD } & \\
\end{array}$ & Overall Mean \pm SD \\
\hline All nurses & $198(100 \%)$ & $62.90 \pm 26.70$ & $47.0 \pm 20.90$ & $51.0 \pm 16.50$ & $53.31 \pm 7.20$ \\
\hline $\begin{array}{l}\text { Health region: } \\
\text { North } \\
\text { South } \\
\text { West } \\
\text { Central } \\
p \text {-value }\end{array}$ & $\begin{array}{l}47(23.7 \%) \\
48(24.2 \%) \\
48(24.2 \%) \\
55(27.8 \%)\end{array}$ & $\begin{array}{l}56.52 \pm 7.44 \\
49.16 \pm 27.40 \\
75.41 \pm 20.10 \\
65.09 \pm 25.00 \\
0.003 *\end{array}$ & $\begin{array}{l}48.92 \pm 0.15 \\
38.19 \pm 25.10 \\
54.39 \pm 16.80 \\
50.10 \pm 18.70 \\
0.136\end{array}$ & $\begin{array}{l}45.18 \pm 15.99 \\
44.61 \pm 20.19 \\
57.23 \pm 10.90 \\
56.57 \pm 13.88 \\
0.000^{*}\end{array}$ & $\begin{array}{l}50.23 \pm 17.31 \\
43.98 \pm 20.48 \\
62.34 \pm 9.76 \\
57.25 \pm 14.29 \\
0.000 *\end{array}$ \\
\hline $\begin{array}{l}\text { Gender } \\
\text { Men } \\
\text { Women } \\
p \text { value }\end{array}$ & $\begin{array}{l}33(17.1 \%) \\
160(82.9)\end{array}$ & $\begin{array}{l}63.63 \pm 19.50 \\
62.75 \pm 27.50 \\
0.857\end{array}$ & $\begin{array}{l}46.80 \pm 22.50 \\
49.16 \pm 20.90 \\
0.547\end{array}$ & $\begin{array}{l}50.08 \pm 18.19 \\
51.83 \pm 16.13 \\
0.580\end{array}$ & $\begin{array}{l}53.50 \pm 16.59 \\
54.58 \pm 16.76 \\
0.737\end{array}$ \\
\hline $\begin{array}{l}\text { Age Group: } \\
\leq 25 \text { years }\end{array}$ & $36(18.3 \%)$ & $62.27 \pm 25.10$ & $48.45 \pm 21.40$ & $51.47 \pm 15.99$ & $54.28 \pm 17.84$ \\
\hline
\end{tabular}




\begin{tabular}{|c|c|c|c|c|c|}
\hline $\begin{array}{l}>31-35 \\
>36 \text { years } \\
p \text { value }\end{array}$ & $\begin{array}{l}92(46.7 \%) \\
69(35.2 \%)\end{array}$ & $\begin{array}{l}57.82 \pm 28.40 \\
66.95 \pm 24.40 \\
0.463 \\
\end{array}$ & $\begin{array}{l}44.93 \pm 21.40 \\
52.33 \pm 18.40 \\
0.381\end{array}$ & $\begin{array}{l}48.97 \pm 17.20 \\
54.13 \pm 15.65 \\
0.449\end{array}$ & $\begin{array}{l}50.57 \pm 18.11 \\
57.80 \pm 14.45 \\
0.327\end{array}$ \\
\hline $\begin{array}{l}\text { Education: } \\
\text { Practical nurse } \\
\text { Registered nurse } \\
p \text { value }\end{array}$ & $\begin{array}{l}63(32.1 \%) \\
133(67.9)\end{array}$ & $\begin{array}{l}58.09 \pm 25.33 \\
63.90 \pm 27.13 \\
0.154\end{array}$ & $\begin{array}{l}45.50 \pm 17.48 \\
49.71 \pm 21.99 \\
0.185\end{array}$ & $\begin{array}{l}48.64 \pm 15.17 \\
52.59 \pm 17.65 \\
0.119\end{array}$ & $\begin{array}{l}49.50 \pm 15.44 \\
55.40 \pm 17.61 \\
0.074\end{array}$ \\
\hline $\begin{array}{l}\text { Years } \\
\text { experience: } \\
\leq 5 \\
6-10 \\
11-15 \\
\geq 16 \\
\text { p-value }\end{array}$ & $\begin{array}{l}55(27.9 \%) \\
58(29.4 \%) \\
33(16.8 \%) \\
51(25.9 \%)\end{array}$ & $\begin{array}{l}73.81 \pm 18.8 \\
55.86 \pm 27.47 \\
49.09 \pm 30.45 \\
64.70 \pm 24.19 \\
0.059\end{array}$ & $\begin{array}{l}54.74 \pm 21.15 \\
45.02 \pm 20.33 \\
37.37 \pm 17.96 \\
51.42 \pm 20.12 \\
0.257\end{array}$ & $\begin{array}{l}55.18 \pm 16.65 \\
48.88 \pm 16.46 \\
47.24 \pm 14.21 \\
52.02 \pm 17.50 \\
0.323\end{array}$ & $\begin{array}{l}61.25 \pm 14.24 \\
49.92 \pm 17.63 \\
44.57 \pm 17.36 \\
56.05 \pm 15.50 \\
0.080\end{array}$ \\
\hline
\end{tabular}

Table 2: Nurses' therapeutic nutrition knowledge and demographic characteristics $(\mathrm{N}=198)$.

*Means are significantly different at $p$ value $<0.05$ by students'-test.

Overweight nurses had the highest knowledge score 57.87\% \pm 15.94 , those who were living with diabetes $(40.10 \% \pm 16.74) \mathrm{p}=.000$. Nurses with followed by those at recommended weight $(52.08 \% \pm 17.19)$ and lastly, by no self-reported diagnosis had significantly higher nutrition and diabetes those who are underweight $(33.0 \% \pm 10.41)$.

scores $63.86 \pm 26.07$ than nurses who had diabetes $43.00 \pm 26.96 \mathrm{p}=0.001$.

Nurses who had no prior diabetes diagnosis had significantly higher overall knowledge scores, higher nutrition and diabetes scores, higher nutrition and obesity scores than nurses who reported diabetes diagnoses (Table 3). Nurses who did not report a diagnosis of diabetes had a significantly higher overall mean knowledge scores $(55.24 \% \pm 16.67)$ than

Nurses with no self-reported diabetes diagnosis had significantly higher nutrition and obesity knowledge scores $49.55 \pm 20.80$ than those who did not have diabetes $35.50 \pm 18.24 \mathrm{p}=0.004$. Nurses with no self-reported diabetes diagnosis had significantly higher nutrition and cardiovascular disease knowledge scores $53.30 \pm 15.70$ than those who did not have diabetes $47.76 \pm 20.63 \mathrm{p}=0.007$.

\begin{tabular}{|c|c|c|c|c|c|}
\hline $\begin{array}{l}\text { Self-reported weight } \\
\text { status and diagnoses }\end{array}$ & Nurses N (\%) & $\begin{array}{l}\text { Nutrition and } \\
\text { diabetes } \\
\text { knowledge } \\
\text { Mean } \pm \text { SD }\end{array}$ & $\begin{array}{l}\text { Nutrition and } \\
\text { obesity } \\
\text { knowledge } \\
\text { Mean } \pm \text { SD }\end{array}$ & $\begin{array}{l}\text { Nutrition and } \\
\text { cardiovascular } \\
\text { disease knowledge } \\
\text { Mean } \pm \text { SD }\end{array}$ & $\begin{array}{l}\text { Overall Mean } \\
\text { (SD) }\end{array}$ \\
\hline $\begin{array}{l}\text { Weight status: } \\
\text { Recommended weight } \\
\text { Overweight } \\
\text { Underweight } \\
\text { p-value }\end{array}$ & $\begin{array}{l}86(44.1 \%) \\
95(48.7 \%) \\
14(7.2 \%)\end{array}$ & $\begin{array}{l}57.90 \pm 27.09 \\
70.10 \pm 22.76 \\
25.17 \pm 14.52 \\
0.059\end{array}$ & $\begin{array}{l}47.93 \pm 21.68 \\
50.50 \pm 20.74 \\
32.53 \pm 16.57 \\
0.257\end{array}$ & $\begin{array}{l}50.41 \pm 15.27 \\
53.00 \pm 16.57 \\
40.75 \pm 13.54 \\
0.510 \\
\end{array}$ & $\begin{array}{l}52.08 \pm 17.19 \\
57.87 \pm 15.94 \\
33.00 \pm 10.41 \\
0.275\end{array}$ \\
\hline $\begin{array}{l}\text { Diabetes diagnosis: } \\
\text { No diabetes } \\
\text { Diabetes } \\
\text { p-value }\end{array}$ & $\begin{array}{l}176(89.8 \%) \\
20(10.2 \%)\end{array}$ & $\begin{array}{l}63.86 \pm 26.07 \\
43.00 \pm 26.96 \\
0.001 *\end{array}$ & $\begin{array}{l}49.55 \pm 20.80 \\
35.50 \pm 18.24 \\
0.004 *\end{array}$ & $\begin{array}{l}53.30 \pm 15.70 \\
47.76 \pm 20.63 \\
0.007 *\end{array}$ & $\begin{array}{l}55.24 \pm 16.67 \\
40.10 \pm 16.74 \\
0.000^{*}\end{array}$ \\
\hline $\begin{array}{l}\text { Hypertension diagnosis: } \\
\text { No hypertension } \\
\text { Hypertension } \\
\text { p-value }\end{array}$ & $\begin{array}{l}164(83.2 \%) \\
33(16.8 \%)\end{array}$ & $\begin{array}{l}63.17 \pm 26.30 \\
54.54 \pm 28.40 \\
0.091\end{array}$ & $\begin{array}{l}48.70 \pm 20.83 \\
44.78 \pm 21.24 \\
0.317\end{array}$ & $\begin{array}{l}51.50 \pm 16.84 \\
50.08 \pm 18.78 \\
0.653\end{array}$ & $\begin{array}{l}54.48 \pm 16.99 \\
49.80 \pm 18.61 \\
0.155\end{array}$ \\
\hline
\end{tabular}

Table 3: Nurses' therapeutic nutrition knowledge and self- reported diagnosis ( $\mathrm{N}=198)$.

*Means are significantly different at $p$ value $<0.05$ by students't-test.

Nurses who reported no previous hypertension diagnosis had higher overall knowledge scores, higher scores on nutrition and diabetes, nutrition and obesity, or nutrition and cardiovascular diseases than nurses who had been diagnosed with hypertension. However, none of these scores were significantly different.

Overall, the item with the highest correct response rate $(80 \%)$ was that high blood cholesterol increases the incidence of heart disease (Table 4).
This was also the item with the highest correct response rate among cardiovascular disease related questions. The item with the highest mean score among diabetes mellitus related questions was that fruits should be consumed by persons living with diabetes ( $77.3 \%$ response rate). The item with the highest mean score among obesity related questions was that nurses agreed a high fiber diet is recommended for obesity $(62.9 \%$ response rate). 


\begin{tabular}{|c|c|c|c|}
\hline $\begin{array}{l}\text { High blood cholesterol increases the } \\
\text { incidence of heart disease. }\end{array}$ & $\begin{array}{l}\text { A. Agree* } \\
\text { B. Disagree } \\
\text { C. Don't know }\end{array}$ & $\begin{array}{l}156 \\
33 \\
6 \\
\end{array}$ & $\begin{array}{l}80.0 \\
16.9 \\
3.1 \\
\end{array}$ \\
\hline $\begin{array}{l}\text { Fruits should not be consumed by the } \\
\text { diabetic patients. }\end{array}$ & $\begin{array}{l}\text { A. Agree } \\
\text { B. Disagree* } \\
\text { C. Don't Know }\end{array}$ & $\begin{array}{l}35 \\
153 \\
10 \\
\end{array}$ & $\begin{array}{l}17.7 \\
77.3 \\
5.1\end{array}$ \\
\hline $\begin{array}{l}\text { For the treatment of hypertension, a low } \\
\text { sodium diet is routinely recommended. }\end{array}$ & $\begin{array}{l}\text { A. Agree* } \\
\text { B. Disagree } \\
\text { C. Don’t know }\end{array}$ & $\begin{array}{l}152 \\
39 \\
6 \\
\end{array}$ & $\begin{array}{l}77.2 \\
19.8 \\
3.0 \\
\end{array}$ \\
\hline $\begin{array}{l}\text { Omega- } 3 \text { fatty acids such as fish oil help to } \\
\text { reduce hyperlipidaemia }\end{array}$ & $\begin{array}{l}\text { A. Agree* } \\
\text { B. Disagree } \\
\text { C. Don’t know }\end{array}$ & $\begin{array}{l}128 \\
42 \\
25 \\
\end{array}$ & $\begin{array}{l}65.6 \\
21.5 \\
12.8 \\
\end{array}$ \\
\hline $\begin{array}{l}\text { A lower intake of saturated fat decreases the } \\
\text { risk of coronary artery disease. }\end{array}$ & $\begin{array}{l}\text { A. Agree* } \\
\text { B. Disagree } \\
\text { C. Don't know }\end{array}$ & $\begin{array}{l}127 \\
48 \\
20\end{array}$ & $\begin{array}{l}65.1 \\
24.6 \\
10.3\end{array}$ \\
\hline
\end{tabular}

Table 4: Top 5 Most frequently correct responses $(\mathrm{N}=198)$.

The five most frequently incorrect statements are presented in Table 5. Only $8.1 \%$ of nurses knew that decreased total fat intake is recommended for an obese 45-year-old adult who had a blood cholesterol level of 239 $\mathrm{mg} / \mathrm{dl}$. This was the item with the lowest correct response rate among all questions. This was also the item with the highest correct response rate among obesity related questions. Among cardiovascular disease related questions, $19.2 \%$ of nurses agreed that intake of $<300 \mathrm{mg}$ of cholesterol is recommended for a low cholesterol diet - the lowest correct response rate among cardiovascular disease related questions. Among diabetes related questions, $47.7 \%$ (the lowest correct response rate) of nurses knew that sugar-free carbonated beverages should not be used for patients with hypoglycemia.

\begin{tabular}{|c|c|c|c|}
\hline Question & Responses & $\mathrm{N}$ & $\%$ correct \\
\hline $\begin{array}{l}\text { An obese } 45 \text {-year-old adult has a blood } \\
\text { cholesterol level of } 239 \mathrm{mg} / \mathrm{dl} \text {. Which of the } \\
\text { following dietary therapies is the first } \\
\text { recommended? }\end{array}$ & $\begin{array}{l}\text { A. Decrease monounsaturated fat intake } \\
\text { B. Decrease polyunsaturated fat intake } \\
\text { C. Decrease total fat intake* } \\
\text { D. Decrease total cholesterol intake } \\
\text { E. Decrease carbohydrate intake } \\
\text { F. Don't know }\end{array}$ & $\begin{array}{l}23 \\
55 \\
55 \\
37 \\
16 \\
12\end{array}$ & $\begin{array}{l}11.6 \\
27.8 \\
8.1 \\
18.7 \\
27.7 \\
6.1\end{array}$ \\
\hline $\begin{array}{l}\text { Intake of less than } \mathrm{mg} \text { of cholesterol is } \\
\text { recommended for a low-cholesterol diet. }\end{array}$ & $\begin{array}{l}\text { A. } 100 \\
\text { B. } 200 \\
\text { C. } 300^{*} \\
\text { D. } 400 \\
\text { E. } 500 \\
\text { F. Don't know } \\
\end{array}$ & $\begin{array}{l}49 \\
62 \\
38 \\
3 \\
4 \\
41 \\
\end{array}$ & $\begin{array}{l}24.9 \\
31.5 \\
19.2 \\
1.5 \\
2.2 \\
20.7 \\
\end{array}$ \\
\hline $\begin{array}{l}\text { Which of the following foods is not a source } \\
\text { of cholesterol }\end{array}$ & $\begin{array}{l}\text { A. Meat } \\
\text { B. Egg } \\
\text { C. Margarine* } \\
\text { D. Milk } \\
\text { E. Fish } \\
\text { F. Don't know }\end{array}$ & $\begin{array}{l}20 \\
2 \\
41 \\
14 \\
106 \\
13 \\
\end{array}$ & $\begin{array}{l}2.2 \\
1.0 \\
29.0 \\
7.1 \\
54.1 \\
6.6 \\
\end{array}$ \\
\hline $\begin{array}{l}\text { The dietary therapy which is not } \\
\text { recommended for preventing coronary } \\
\text { artery disease is: }\end{array}$ & $\begin{array}{l}\text { A. Replace iso-calories of unsaturated } \\
\text { by saturated fatty acids* } \\
\text { B. Consume complex carbohydrates as } \\
\text { the majority of daily caloric intake } \\
\text { C. Reduce food high in cholesterol } \\
\text { D. Decrease total fat intake } \\
\text { E. Increase fruits and non-starch } \\
\text { vegetables } \\
\text { F. Don't know }\end{array}$ & $\begin{array}{l}58 \\
45 \\
52 \\
10 \\
10 \\
18\end{array}$ & $\begin{array}{l}30.1 \\
23.3 \\
26.9 \\
5.2 \\
5.2 \\
9.3\end{array}$ \\
\hline
\end{tabular}

Table 5: The five most frequently incorrect statements.

The profile of a knowledgeable nurse was a female registered nurse from the Western region who had less than five years' experience, who was more than 36 years old, who was overweight but had not been diagnosed with neither diabetes nor hypertension. The profile of a less than knowledgeable nurse was a male licensed practical nurse from the South region, who had 11-15 years' experience, who was more than 35 years old, who had been diagnosed with diabetes mellitus and hypertension, and who was underweight.

\section{Discussion}

As has been demonstrated in earlier studies, Belizean nurses have low levels of therapeutic nutrition knowledge (mean score $=53.30 \% \pm 17.20$ ). The two most recently reported mean knowledge scores were for 
Jordanian nurses $58.8 \% \pm 13.2$ in 2013 [17], and for Ghanaian nurses $44.9 \% \pm 3.17$ in 2015 [19]. The only published studies which have shown higher levels of nurses' nutrition knowledge were from Australia $60 \% \pm 8.4$ in 2005 [15], and the United States of America 65\% \pm 11 in 2001 [20]. Nurses in Belize's West region had a significantly higher overall mean, higher mean knowledge scores for nutrition and diabetes; and for nutrition and cardiovascular diseases; compared to nurses in other regions. The West region of Belize is the site of the capital city, Belmopan.

No other published studies have assessed the relationship between nurses' nutrition knowledge scores and their weight status and previous diagnosis of diabetes and hypertension. Nurses who had not been diagnosed with diabetes had significantly higher knowledge scores overall, in nutrition and diabetes, nutrition and cardiovascular diseases, and in nutrition and obesity. This was a surprising, if not troubling, discovery. Ideally, nurses who have been diagnosed with a non-communicable disease should not only be knowledgeable about disease management but they should have achieved success in managing the condition. Since Afro-Trinidadian adolescents and Jamaica adults have been shown to view overweight and obesity are viewed favorably as signs of happiness or wealth [22-24], it would be important for future research to compare Caribbean nurses' actual and perceived weight status to nutrition knowledge. Future research could address the extent to which nurses' nutrition knowledge is related to glycemic control and blood pressure levels.

These data did not confirm the data from one earlier study which showed a direct relationship between knowledge scores and age, and years of nursing experience. Using a different 48-item instrument, Australian nurses who were more than 36 years of age had statistically significant higher nutrition knowledge scores $65.2 \%$ than nurses who were 35 years or younger $52.5 \% \mathrm{p}=0.004$ [15]. Similarly, Australian nurses with more than 10 years of experience had a statistically significant higher knowledge score than nurses with 10 years or less experience $\mathrm{p}=0.024$, although actual scores were not stated.

Earlier studies have reported inconsistencies in the relationship between nurses' knowledge and educational preparation. In Australia, nurses with a Diploma or General Nurse Training (less than four years of preparation) had a statistically significantly higher knowledge score $63.5 \%$, than nurses with a four-year degree $54.6 \% \mathrm{p}=0.029$ [15]. However, among Turkish nurses, knowledge scores were significantly higher as educational preparation increased $\mathrm{p}<0.001$ (16). Approximately $45.2 \%$ graduate nurses had "good" (13-16 points) mean scores, 39.3\% undergraduate nurses had "adequate" (9-12 points) mean scores, and $15.2 \%$ of secondary education nurses had "inadequate" ( $\leq 8$ points) mean scores.

Earlier studies have also identified statistically significant but an inconsistent relationship between nurses' nutrition knowledge and gender. In Jordan, nutrition knowledge on cardiovascular diseases was significantly higher for female nurses $56.6 \%$, compared to male nurses $51.9 \% \mathrm{p}=0.04$ [17]. However, in Ghana, male nurses had a significantly higher nutrition knowledge score $12.93 \pm 3.10$ than females $11.34 \pm 3.05$ $\mathrm{p}=0.003$ using a 21 -item instrument [19]. The variation in results from different studies may be related to the different types of questionnaires used in the studies, the size of the sample, differences in the scope of practice of the nurses among countries, and variation in the nursing school curriculum among different countries.

The low level of therapeutic nutrition knowledge of Belizean nurses $53.30 \% \pm 17.20$ is similar to levels described in Jordan and Turkey, but lower than studies in the USA $65 \% \pm 11.0$ [20], and Australia $60 \% \pm 8.4$ [15].

One study limitation is that completing questionnaires during work hours may have influenced nurses' interpretation and responses to questions. These data were derived from a convenience sample and should therefore not be generalized to all Belizean nurses. Future research may need to quantify the extent to which nurses provide nutrition services - screening, counseling, and monitoring--to hospitalized patients.

\section{Conclusion}

This study showed that nurses in Belize have suboptimal level of therapeutic nutrition knowledge $(53.30 \% \pm 17.20)$. In small countries such as Belize where nutrition professionals are uncommon, and there is no national plan to increase the number of trained dietitians and nutritionists, it is even more important to ensure that nurses have adequate therapeutic nutrition knowledge. Nurses need to have access and to be rewarded for continuing education in therapeutic nutrition as part of their licensing requirements. Mandatory continuing education in nutrition could be part of nurses' licensing requirements. To improve compliance, nurses need to be consulted on their preferences for learning modalities such as elearning and Medscape [25].

\section{References}

1. Global Nutrition Report [Internet]. c2014-2020 [cited 2020 August 10]. Country Overview [about 8 screens]. Available from: https://globalnutritionreport.org/resources/nutritionprofiles/latin-america-and-caribbean/central-america/belize/ 2019 Belize Nutrition Profile.

2. Pan American Health Organization [Internet]. Washington D.C.: c1902-2017 [cited 2020 Aug 12]. Country Report; [about 8 screens]. Available from:https://www.paho.org/salud-en-lasamericas-2017/?page_id=91. Country Report: Belize.

3. Hambleton IR, Narayan KMV. (2014). Diabetes in North America and The Caribbean: An update. Diabetes Res Clin Pract. 2014 Feb 8;103(2):223-30. doi: 10.1016/j.diabres.2013.11.009. Epub. Pub Med PMID: 24321468.

4. Kaiser MJ, Bauer JM, Rämsch C, Uter W, Guigoz Y, Cederholm T, Maggio, M. (2010) .Frequency of malnutrition in older adults: A multinational perspective using the mini nutritional assessment. J Am Geriatr Soc. Sep 10;58(9):1734-1738. doi:10.1111/j.15325415.2010.03016.x. PubMed PubMed PMID: 20863332.

5. 5.Tappenden KA, Quatrara B, Parkhurst ML, Malone AM, Fanjiang G, Ziegler TR (2013). Critical role of nutrition in improving quality of care: An interdisciplinary call to action to address adult hospital malnutrition. JPEN J Parenter Enteral Nutr. 2013 Jul;37(4):482-97. doi: 10.1177/0148607113484066. Epub 2013 Jun 4. DOI: 10.1177/0148607113484066. Pub Med PMID: 23736864.

6. United States Bureau of Labor. Statistics Occupational Outlook Handbook 2010-2011. 2011. Labor Statistics Bureau Bulletin 2800.

7. Xiaoyue X, Parker D, Ferguson C, Hickman L (2017). Where is the nurse in nutritional care?. Contemporary Nurse; 53:3, doi: 10.1080/10376178.2017.1370782, 267-270.

8. Jefferies D, Johnson M, Ravens J (2011). Nurturing and nourishing: The nurses' role in nutritional care. J Clin Nurs. (34):317-30. doi: 10.1111/j.1365-2702.2010.03502.x. PubMed PMID: 21219515.

9. McClinchy J, Williams J, Gordon L, Cairns M, Fairey G (2015). Dietary Advice and Collaborative Working: Do Pharmacists and Allied Health Professionals Other Than Dietitians Have a Role? Healthcare (Basel); 3(1):64-77. doi:10.3390/healthcare3010064. PubMed PMID:27417748; PubMed Central PMCID: PMC4934524.

10. Ministry of Health New South Wales. (2011). Nutrition care. Ministry of Health.

11. Varley E, Maureen M. An implementation guide and toolkit for making every contact count: Using every opportunity to achieve health and wellbeing. 
12. DiMaria-Ghalili RA, Mirtallo JM, Tobin BW, Hark L, Van Horn L, Palmer CA (2014). Challenges and opportunities for nutrition education and training in the health care professions: intraprofessional and interprofessional call to action. Am J Clin Nutr.;99(5 Suppl):1184S-1193S. doi: 10.3945/ajcn.113.073536. Pub Med PMID: 24646823: PubMed Central PMCID: PMC3985220.

13. The Academy of Nutrition and Dietetics. Licensure and Professional Regulation of Dietitians.

14. Kgaphola MS, Wodarski LA. Garrison, MEB (2003). Nutrition knowledge of clinic nurses in Lebowa, South Africa: implications for nutrition services delivery. Journal of Human Nutrition and Dietetics;10(5):295-303.

15. Schaller C, James EL (2005). The nutritional knowledge of Australian nurses. Nurse Educ Today.; 25(5): PubMed PMID: 15946775. doi:10.1016/j.nedt.2005.04.002, 405-412.

16. Ozcelyk A, Surucuoglu M, Akan L (2007). Nutrition knowlege level of nurses in Turkey: Ankara as an example. Parkistan Journal of Social Sciences. 4(3):485-489.

17. Al-Shwaiyat NM, Sinjillawi AB, Al-Rethaiaa AS, Fahmy AA, AlSaraireh RM, Aqel MM, Al-Hajjaj SM, Alaa S. Al-Sbou (2013). Assessment of Therapeutic Nutritional Knowledge of Jordanian Nurses, International Journal of Nutrition and Food Sciences;142. doi: 10.11648/j.ijnfs.20130203.18.

18. Park KA, Cho WI, Song KJ, Lee YS, Sung IS, Choi-Kwon SM
(2011). Assessment of nurses' nutritional knowledge regarding therapeutic diet regimens. Nurse Educ Today;31(2):192-197.

19. Mogre V, Ansah, GA, Marfo DN, Garti HA (2015). Assessing nurses' knowledge levels in the nutritional management of diabetes. International Journal of Africa Nursing Sciences; 40. https://doi.org/10.1016/j.ijans.2015.07.003

20. Crogan NL, Shultz JA, Massey LK (2001). Nutrition knowledge of nurses in long-term care facilities. $J$ Contin Educ Nurs;32(4):171-176.

21. Statistical Packages for the Social Sciences 25.0. Cary, NC: International Business Machines. 2020.

22. Hoyos MD, Clarke H (1987). Concepts of obesity in family practice. West Ind Med $\mathrm{J} ; 36(2): 95-98$.

23. Chutkan ME, Meeks Gardner JM, Wilks RJ (2001). Concepts of obesity among outpatients of a Jamaican Hospital. Cajanus.;34 (3):127-134.

24. Simeon DT, Rattan RD, Panchoo K, Kungeesingh KV, Ali AC, Abdool PS (2003). Body image of adolescents in a multi-ethnic Caribbean population. Eur J Clin Nutr; 57(1):157-162.

25. Herriot AM, Bishop JA, Kelly M, Murphy M, Truby H (2003). Evaluation of a computer assisted instruction resource in nursing education. Nurse Education Today. 2003;23 (7):537-545.

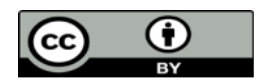

This work is licensed under Creative Commons Attribution 4.0 License
Ready to submit your research? Choose Auctores and benefit from:

* fast, convenient online submission

* rigorous peer review by experienced research in your field

* rapid publication on acceptance

* authors retain copyrights

* unique DOI for all articles

* immediate, unrestricted online access

At Auctores, research is always in progress.

Learn more www.auctoresonline.org/journals/new-medical-innovationsand-research 